\title{
Murray Gell-Mann
}

\section{Nobel Prize for Physics 1969}

\section{A. Radicati di Brozolo}

To review the work of Gell-Mann comes very close to analyzing the major achievements of particle physics in the post-parity era.

The Leitmotiv of Gell-Mann's whole work has been the search for the ordering principles and the deep regularities in the subnuclear world. This world, essentially unknown twenty years ago, has shown the same complexity and variety of phenomena as the atomic and molecular world. The key to the understanding of the latter had been Bohr's model: Gell-Mann's SU (3) scheme with its subsequent extensions seems to play a similar role in the subnuclear domain.

In his search for the order underlying the complexity of elementary particle physics, Gell-Mann has focused his attention on the internal variables which characterize the elementary particles (I will still use the word "elementary" for lack of a better one) and their behaviour with respect to the different interactions. Already in 1953, Gell-Mann, generalizing some previous work of Nishijima and his coworkers, established an empirical relation between the charge, the third component of the isotopic spin, and a new quantum number - the hypercharge. So, by assuming that the hypercharge, like the hadronic electromagnetic charge, is conserved by all interactions except the weak, the multiplicity of the strange particles and their decay schemes received a simple interpretation.

It was eight years later that GellMann was able to show that the approximate conservation of the two additive quantum numbers, the hadronic charge and the hypercharge, were the result of the approximate invariance of the strong interactions under the eight dimensional Lie group SU (3). The difficulty of showing the existence of this higher symmetry lay in its approximate character which makes the real world highly asymmetrical. By exploiting the simple behaviour of the symmetry breaking term, Gell-Mann was able to predict the position of several still unknown hadronic states. Their subsequent experimental discovery provided, soon afterwards, a convincing proof of the exactness of the theory.

The SU (3) symmetry not only gave a meaningful classification for the hadronic states, it also led to a unified picture of the two parts of the weak hadronic current and their selection rules. This was made possible by another piece of work that Gell-Mann had done in 1957 in collaboration with Feynman. In that fundamental paper, the two authors had shown the connection between the generators of the strong interaction symmetry and the currents coupled to the electromagnetic and to the lepton fields.

This idea, which Gell-Mann soon afterwards extended to SU (3), has become one of the corner stones of elementary particle physics and has given us an important clue to the understanding of the relations between the fundamental interactions.

Right from the beginning of his work on SU (3), Gell-Mann pointed out that the connection between the currents and the generators of the symmetry group would naturally lead, in order to include the axial currents, to an extension of the symmetry scheme from SU (3) to SU (3) $\times$ SU (3). With the help of a simple model - the quark model - he analyzed the algebra generated by the integrals over the time components of the vector and axial vector currents and pointed out some of its implications. Several important relations were soon afterwards derived from this algebra by Adler, Weissberger, Weinberg and other people. Recently Gell-Mann and his coworkers have shown that not only the SU (3) $\times$ SU (3) algebra is important but that the symmetry group itself is likely to represent a meaningful approximation to the whole hadronic physics.

Perhaps one day we will understand the origin of SU (3), of SU (3) $X S U(3)$ and why the quark model works, just as Bohr's model was understood in the late twenties after the discovery of quantum mechanics. Then the importance of Gell-Mann's contributions to the unravelling of the mysteries of particle physics will be even more deeply appreciated.

\section{International Computer Program Library}

An international physics computer program library has recently been started at the Queen's University of Belfast, Northern Ireland, with the aid of a grant from the Science Research Council, London.

The programs, written in an International Source Language, are contributed by leading physicists from Laboratories and Institutes all over the world. Detailed descriptions of the programs are given in a new Journal called "Computer Physics Communications" published by North-Holland Publishing Company, Amsterdam, and the library is stored and indexed on magnetic tape and disc files on the Queen's University ICL 1907. Retrieval programs are being written to allow rapid access to information on any program in the library.

The Belfast library distributes programs on request to two classes of user. Institutes and Laboratories can become subscribers and receive, on magnetic tape, a copy of every program that comes into the library. An individual physicist can request a copy of a single program or a few programs. A charge is made for these services to cover handling, but the library is non-profit making. It is hoped that the publication and distribution of programs, as well as reducing the costly duplication of programming effort, will enable authors to obtain appropriate recognition for their work, and will promote rapid advance in the use of computers and computing techniques amongst the physics community.

As the number of programs in the Belfast library, and the need for their rapid distribution, grow, it is intended to start other libraries forming a network in Europe and in the USA. Several institutes have already agreed to collaborate in this way and will be supplied from the central library at Belfast.

Further information, including details on how to become a subscriber to the Belfast library, can be obtained on request from Professor P.G. Burke, Queen's University, Belfast. 